\title{
NHANES III: Wann kardiovaskuläre Risikofaktoren aggressiv behandeln?
}

Hintergrund: Bei Personen mit Typ-2-Diabetes kann durch die Senkung von Lowdensity lipoprotein cholesterol (LDL-C) und Blutdruck das Risiko für kardiovaskuläre Erkrankungen reduziert werden. Allerdings brachte eine Analyse im Rahmen der NHANES-III-Studie zutage, dass Nutzen und Risiken einer aggressiven Modifikation kardiovaskulärer Risikofaktoren interindividuell stark variieren.

Methode: Im Rahmen der National Health and Nutrition Examination Survey III (NHANES III)-Studie wurden Personen mit Typ-2-Diabetes im Alter von 30 bis 75 Jahren ausgewählt. Soweit erforderlich wurde die Therapie intensiviert mit einem Ziel-LDL-C von $100 \mathrm{mg} / \mathrm{dL}$ und einem Ziel-Blutdruck von 130/80 mmHg. Eine Anpassung der Statindosis erfolgte bis zu fünfmal und die der Dosis der Anti- hypertensiva bis zu achtmal. Die Endpunkte wurden anhand des MarkovModells ermittelt.

Ergebnis: Durch das Erreichen der Zielwerte wurde ein Zuwachs von 1,5 (LDL-C) bzw. 1,35 (Blutdruck) qualitätsadjustierten Lebensjahren (QALY) bezogen auf den mit der Behandlung assoziierten Nutzen beobachtet. Die Zahl der QALYs reduzierte sich auf 1,42 bzw. 1,16, sofern auch der mit der Behandlung assoziierte negative Nutzen berücksichtigt wurde. Der Nutzen war hauptsächlich auf die ersten Schritte der Therapieintensivierung sowie auf die Therapie von Hochrisikopatienten zurückzuführen. Bei Personen mit geringem kardiovaskulären Risiko war eine Intensivierung der Therapie über den ersten Schritt (LDL-C) bzw. den dritten Schritt (Blutdruck) hinaus mit entweder

einem nur geringen Nutzen oder nega-
tiven Nutzen assoziiert.
Folgerung: Bei Personen mit Typ-2-Dia-
betes variieren Nutzen und Risiken einer
Senkung von LDL-C und Blutdruck von
Person zu Person sehr stark, wobei sich
der Nutzen mit steigendem kardiovasku-
lären Risiko der Person erhöht. Daher
sollte bei jedem Menschen ein individu-
eller Therapieansatz gewählt werden, um
den Nutzen einer Therapie zu maximie-
ren, so die Autoren.
Dr. med. Winfried Keuthage, Münster
Quelle: Timbie JW et al. Variation in the Net
Benefit of Aggressive Cardiovascular Risk Factor
With Diabetes Mellitus. Arch Intern Med 2010;
170: 1037-1044

